\title{
Quasi-fractal PBG structures for Multi-Beam Devices
}

\author{
V. A. Tsarev ${ }^{1}$, D. A. Nesterov ${ }^{2}$, P. D. Shalaev ${ }^{3}$ \\ ${ }^{1}$ Yuri Gagarin Saratov State Technical University, Saratov, Russia, tsarev_va@mail.ru \\ ${ }^{2}$ Yuri Gagarin Saratov State Technical University, Saratov, Russia, enlucioro@gmail.com \\ ${ }^{3}$ AO “NPP “Almaz”, Saratov, Russia, p.d.shalaev@yandex.ru
}

Over the last years, the great attention of microwave vacuum tube and accelerator researchers therefore drawn to so-called photonic crystal resonator cavities, formed by a defect lattice (photonic band gap structure-PBG)[1-4].

Very strict requirements applied to the resonator systems used in such devices. They must have a high characteristic impedance $\rho=R / Q_{0}$ and increased (compared to the operating wavelength) dimensions. In addition, they must have a rigid and technological structure having a high unloaded Q-factor and a good heat-dissipating ability.

In the defect area it is appeared local electromagnetic waves (defect modes) similar to electromagnetic waves of ordinary cavity resonators.

The advantages of PBG are increased overall dimensions, high unloaded Q-factor, comparative simplicity of engineering implementation, as well as the possibility to suppress undesirable (parasitic oscillation).

Due to these advantages, the metal photonic band gap structure has potentialities in the areas of highenergy accelerators, multi-beams klystrons.

Up to the present moment, it was studied only one gap single defects and multiple defects of PBG for single-beam and multi-beam klystrons $[5,6]$. This did not allow us to realize a large gain factor, a high efficiency and a wide band in such devices.

This paper presents the results of $3 \mathrm{D}$ computer simulations of a double-gap PBG resonator with enhanced impedance of interaction.

The transformation of the defect zone from twodimensional to three-dimensional was accomplished by inserting an additional $\mathrm{X}$-shaped quasi-fractal resonance element in the cross section in the central part of the interaction region (fig. 1). This transformation was performed by inserting an additional X-shaped quasi-fractal resonance element in transverse section in central part of the interaction area.

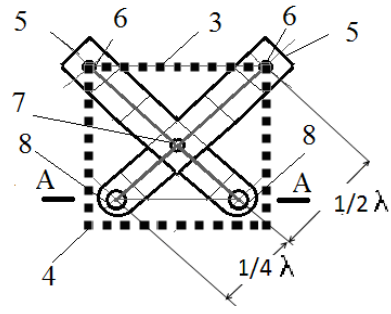

a)

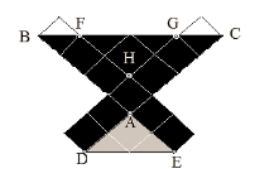

b)

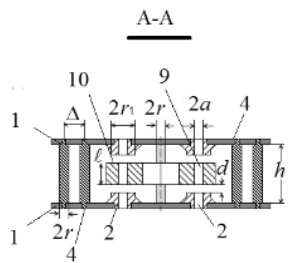

c)
Fig. 1. The structure of the resonator: a) fractal scheme, b) top view, c) transverse section
The interaction area is confined by side covers 1 with circular holes 2 for transition of two electron beams in longitudinal direction and in transverse direction by a conditional square 3 that defines boundary of the longitudinal rods location 4 , which restrict two-dimensional defect area. The resonance rods 5, forming the additional X-shaped resonance element, are oriented on the diagonal of the conditional square in the two-dimensional photonic crystal. This element consisted of two similar triangles (ADF and $\mathrm{ABC}$ ) with cutout in them (FGH and ADE), which are also scale lines of the initial triangles. The drawn figure is similar to Serpinski triangle fractal [7]. In the center of diagonal intersection at the distance $l_{1}=\lambda / 2$ from electric contact place between resonance rods with longitudinal rods 6 is located a central longitudinal metal rod 7, having electric contact with side covers. At the free ends of the resonance rods it is made circular holes 8 for electron beams transition that are coaxial with circular holes on side covers. The full length of both resonance rods is equal $l_{2}=3 / 4 \lambda$, where $\lambda$ is TEM - mode operating wavelength. As a result of these transformations it is formed two double high-frequency gaps. The lowest frequency modes in three-dimensional photonic band gap are quasi-TEM modes, which electric и magnetic fields have opposite directions in each gap ( $\pi$-mode). However, for operation in millimeter and sub-millimeter wavelength ranges in-phase modes similar to $\mathrm{TM}_{110}$ and $\mathrm{TM}_{210}$ in ordinary prismatic resonant cavities is of the greatest interest.

\section{Simulation results}

We have designed a quasi-fractal double-gap cavity based on PBG structure. 3D-models of studied resonance system are illustrated in table.

Table. Computer models of studied PBG structure

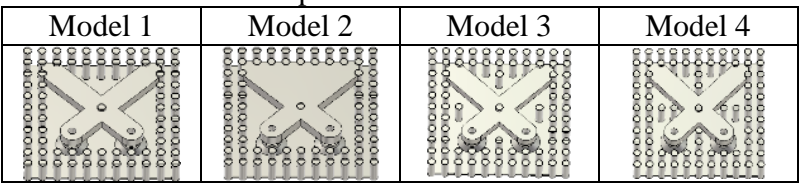

It had the following dimensions: lattice depth 10 $\mathrm{mm}$, metal rods diameter $2 \mathrm{~mm}$, spacing between the rods, $3.7 \mathrm{~mm}$, drift tube radius $1.0 \mathrm{~mm}$; gap length 1.5 $\mathrm{mm}$; resonance rods thickness in along-track beam direction $3 \mathrm{~mm}$. It was studied the influence of number $n$ additional longitudinal rods that are inserted into 3D defect area, at a frequency of indicated modes. During the simulation, it is also determined characteristic impedance (shunt impedance, fig.2) and unloaded Q-factor cavity (fig.3). 


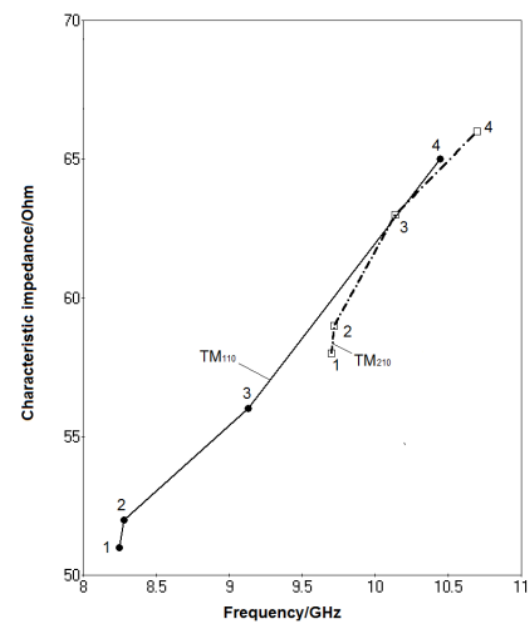

Fig. 2. HOM $R / Q_{0}$ as a function of frequency. Numbers indicate the model numbers indicated in table.

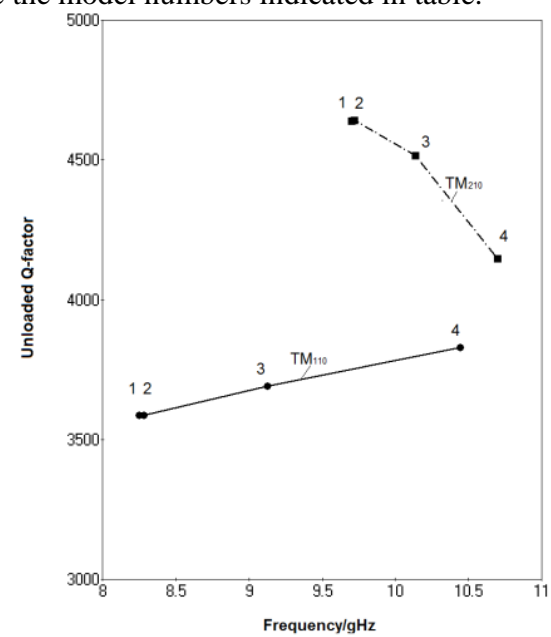

Fig. 3. HOM $Q_{0}$ as a function of frequency. Numbers indicate the model numbers indicated in table.

From fig. 2 it is clear that increasing numbers of rods in the cavity volume leads to characteristic impedance increasing of both $\mathrm{TM}_{110}$ (1.3 times) and $\mathrm{TM}_{210}$ modes (1.2 times). The dependence of unloaded Q-factor on rod numbers (fig. 3) for $\mathrm{TM}_{110}$ mode has linear increasing form while for $\mathrm{TM}_{210}$ mode Qfactor it falls. Considering both dependence we can conclude: stunt impedance linearly grows with the rods number for $\mathrm{TM}_{110}$ and has a maximum at a number of rods $n=2$ (fig. 4). The dependence of stunt impedance for $\mathrm{TM}_{210}$ on frequency has the parabola form, a maximum is at $n=1$. Having said so this mode has higher stunt impedance values then at $\mathrm{TM}_{110}$ mode for any $n$.

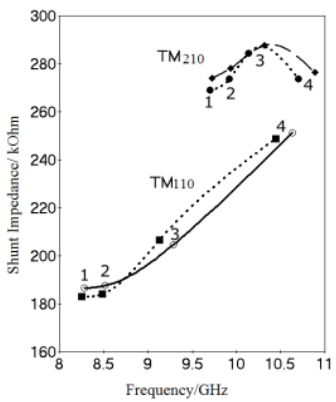

Fig. 4. Shunt impedance for HOM as a function of frequency. Numbers indicate the model numbers indicated in table. Dotted lines represent cavities with the rods, dashed and solid lines represent cavities with solid walls

The presence of the rods in quasi-fractal cavity models leads to frequency decreasing as compared with cavity models with solid walls.

The coupled quasi-fractal double-gap cavity array as a fractal electrodynamic system that capable to slow down electromagnetic wave considerably can be used for TWTs.

Due to increased stunt impedance, quasi-fractal PBG structures for Multi-Beam Devices has potentialities in the areas of high-energy accelerators, multibeams klystrons, terahertz radiation sources, etc.

\section{References}

1. Ozbay E., Temelkuran B., and Bayindir M. Microwave applications of photonic crystals // Progress In Electromagnetics Research, 2003. Vol. 41, pp. 185-209.

2. Ashutosh, Jain P.K. Design and analysis of metallic photonic band gap cavity for a gyrotron // Journal of Microwaves, Optoelectronics and Electromagnetic Applications. 2012. Vol. 11, № 2. P. 242.

3. Han S.-T., Jeon S.-G., Shin Y.-M., Jang K.-H., So J.K., Kim J.-H., Chang S.-S., Park G.-S. Experimental investigations on miniaturized high-frequency vacuum electron devices // IEEE Trans. Plasma Sci. 2005. Vol. 33, № 2. P. 679.

4. Jeon S.-G., Shin Y.-M., Jang K.-H., Han S.-T., So J.K., Joo Y.-D., Park G.-S. High order mode formation of externally coupled hybrid photonic-band-gap cavity // Appl. Phys. Lett. 2007. Vol. 90, № 2. 021112.

5. Smirnov A. V. et. al. PBG Cavities for Single-Beam and Multi-Beam Electron Devices // Proceedings of Particle Accelerator Conference, 2003, pp. 1153-1155.

6. Xu, Y. and Seviour, Rebecca. Design of Photonic Crystal Klystrons. In: Proceedings of the 1st International Particle Accelerator Conference IPAC 2010. JACoW, Kyoto, Japan, pp. 4002-4004. ISBN 978-92-9083-352-9.

7. Sierpinski triangle // Wikipedia, the free encyclopedia. URL: https://en.wikipedia.org/wiki/Sierpinski_triangle. 06.09.18. 